\title{
Abnormal dilepton yield from local parity breaking in heavy-ion collisions
}

\author{
A.A. Andrianov ${ }^{1,2 *}$, V.A. Andrianov ${ }^{1}$, D. Espriu ${ }^{2,3}$, and X. Planells ${ }^{2}$ \\ 1 V.A.Fock Department of Theoretical Physics, Saint-Petersburg State University, 198504, St.Petersburg, Russia \\ 2 Departament d'Estructura i Constituents de la Matèria and Institut de Ciències del Cosmos (ICCUB), \\ Universitat de Barcelona, Martí i Franquès 1, 08028 Barcelona, Spain and \\ 3 CERN, 1211 Geneva, Switzerland
}

\begin{abstract}
We propose a novel explanation for the dilepton excess observed in heavy ion collisions at invariant masses below $1 \mathrm{GeV}$. We argue that the presence of local parity breaking due to a time-dependent isosinglet and/or isotriplet pseudoscalar condensate substantially modifies the dispersion relation of photons and vector mesons propagating in such a medium, changing the $\rho$ spectral function and resulting in a potentially large excess of $e^{+} e^{-}$and $\mu^{+} \mu^{-}$with respect to the theoretical predictions based in a 'cocktail' of known hadronic processes. Possible signatures to prove or disprove this effect are discussed.
\end{abstract}

PACS numbers: 25.75.-q, 25.75.Cj, 21.65.Jk, 12.40.Vv

During the last decade several experiments in heavy ion collisions have indicated an abnormal yield of lepton pairs with invariant mass $M<1 \mathrm{GeV}$ in the region of small rapidities and moderate transversal momenta [1, 2] (reviewed in $[3,4]$ ). This effect is visible only for collisions that are central or semi-central, and is present for $e^{+} e^{-}$and also for $\mu^{+} \mu^{-}$. From a comparison to $p p$ and $p$-nucleus collisions it has been established beyond doubt that such an enhancement is a nuclear medium effect [3]. For the energies accessible at GSI (of few $\mathrm{GeV}$ per nucleon in HADES experiments [3]) the effect was partially interpreted as being due to enhanced $\eta$ meson production in proton-neutron scattering [5]. For the higher energies accessible at CERN SPS and BNL RHIC (with $\sqrt{s_{N N}}$ ranging from $20 \mathrm{GeV}$ to $200 \mathrm{GeV}$ per nucleon in c.m., in CERES, HELIOS/3, NA60 [1] and PHENIX [2] experiments) the abnormal dilepton yield has not been yet explained satisfactorily by known processes in hadronic physics [3, 4].

Following [2] we divide the range of dilepton invariant masses into high $(M>3.2 \mathrm{GeV})$, low $(M<1.2 \mathrm{GeV})$ and intermediate. The low mass region (LMR) is in turn divided into LMR I with $M<0.3 \mathrm{GeV}$ and LMR II with $0.3 \mathrm{GeV}<M<1.2 \mathrm{GeV}$. In LMR I the enhancement could possibly be explained by modifications of meson properties in nuclear medium [6 6 ] as well as by protonneutron scattering [5]. But in the LMR II the $\rho$ meson, directly via $\pi \pi$ fusion or indirectly through Dalitz processes, largely dominates and the in-medium effects of a dropping mass and/or broadening resonance seem unable to explain the spectacular dilepton enhancement by a 4 to 7 factor, depending on $p_{T}$ and centrality (see [10, 11] for recent attempts).

In this letter we propose a radically different explanation of this enhancement. We suggest that the effect may be a manifestation of local parity breaking (LPB) in colliding nuclei due to the generation of a pseudoscalar, isos-

\footnotetext{
${ }^{*}$ Corresponding author. E-mail: andrianov@icc.ub.edu
}

inglet or neutral isotriplet, condensate whose magnitude depends on the dynamics of the collision. It has been suggested that such a background could be due to the topological charge fluctuations leading to the so-called Chiral Magnetic Effect (CME) 12] studied by lattice QCD simulations [13] and seemingly detected in the STAR and PHENIX experiments at RHIC 14], although the issue is far from being settled. It might be also related to pseudoscalar domain walls [15]. However the fact that the observed dilepton excess is almost absent for peripheral collisions (where the CME should be more visible) and maximized in cental collisions makes us believe that it may be due to the ephemeral formation of a bona-fide thermodynamic phase where parity is broken, a possibility that has been argued for in [16].

It has been shown in 17] that a pseudoscalar field slowly evolving in time drastically changes the electromagnetic properties of the vacuum. In particular an energetic photon propagating in this background may decay on shell into dileptons. This same mechanism extended to vector mesons is proposed here as the source for the abnormal dilepton yield in the LMR, i.e. in the range $M<1.2 \mathrm{GeV}$, for centrality $0 \div 20 \%$ and for moderate $p_{T}<1 \mathrm{GeV}$ [2]. In this letter we will concentrate in the LMR II, and more specifically in the region around the $\rho$ and $\omega$ resonant contribution.

We shall assume that a time dependent but approximately spatially homogeneous background of a pseudoscalar field $a(t)$ is induced at the densities reached in heavy ion collisions and we will define a 4 -vector related, $\zeta_{\mu} \simeq \partial_{\mu} a$, for later use. $a(t)$ could be either isosinglet or isotriplet or even a mixture of the two, but detailed calculations will be presented for the case of isosinglet background only.

The appropriate framework to describe electromagnetic interactions of hadrons at low energies is the Vector Dominance Model (VDM) [7, 18] containing the lightest vector mesons $\rho_{0}$ and $\omega$ in the $S U(2)$ flavor sector. We do not include $\phi$ meson, as its typical mean free path $\sim 40$ fm makes it insensitive to medium effects. Quark-meson 
interactions are described by

$$
\begin{aligned}
& \mathcal{L}_{i n t}=\bar{q} \gamma_{\mu} V^{\mu} q ; \quad V_{\mu} \equiv-e A_{\mu} Q+\frac{1}{2} g_{\omega} \omega_{\mu} \mathbf{I}+g_{\rho} \rho_{\mu} \frac{\tau_{3}}{2}, \\
& \left(V_{\mu, a}\right) \equiv\left(A_{\mu}, \omega_{\mu}, \rho_{\mu} \equiv\left(\rho_{0}\right)_{\mu}\right),
\end{aligned}
$$

where $Q=\frac{\tau_{3}}{2}+\frac{1}{6} \mathbf{I}, g_{\omega} \simeq g_{\rho} \equiv g \simeq 6$. These values are extracted from vector meson decays. The Maxwell and mass terms are

$$
\begin{aligned}
& \mathcal{L}_{\text {kin }}=-\frac{1}{4}\left(F_{\mu \nu} F^{\mu \nu}+\omega_{\mu \nu} \omega^{\mu \nu}+\rho_{\mu \nu} \rho^{\mu \nu}\right) \\
& \mathcal{L}_{\text {mass }}=m_{V}^{2} \operatorname{tr}\left(V_{\mu} V^{\mu}\right)=\frac{1}{2} V_{\mu, a} m_{a b}^{2} V_{b}^{\mu}, \\
& m_{a b}^{2}=m_{V}^{2}\left(\begin{array}{ccc}
\frac{10 e^{2}}{9 g^{2}} & -\frac{e}{3 g} & -\frac{e}{g} \\
-\frac{e}{3 g} & 1 & 0 \\
-\frac{e}{g} & 0 & 1
\end{array}\right), \operatorname{det}\left(m^{2}\right)=0,
\end{aligned}
$$

where $m_{V}^{2}=m_{\rho}^{2}=2 g_{\rho}^{2} f_{\pi}^{2} \simeq m_{\omega}^{2}$. This matrix reflects the VMD relations at the quark level [7, 18]. Finally, in a pseudoscalar time-dependent background the Lagrangian contains a parity-odd Chern-Simons (CS) term

$$
\begin{aligned}
& \mathcal{L}_{C S}(k)=-\frac{1}{4} \varepsilon^{\mu \nu \rho \sigma} \operatorname{tr} \hat{\zeta}_{\mu} V_{\nu}(x) V_{\rho \sigma}(x) \\
& =\frac{1}{2} \operatorname{tr} \hat{\zeta} \epsilon_{j k l} V_{j} \partial_{k} V_{l}=\frac{1}{2} \zeta \epsilon_{j k l} V_{j, a} N_{a b} \partial_{k} V_{l, b},
\end{aligned}
$$

which additionally mixes photons and vector mesons due to LPB. For isosinglet pseudoscalar background $e^{2} \hat{\zeta}=$ $\frac{9}{5} \zeta \mathbf{I}$, and the mixing matrix reads

$$
N_{a b} \simeq\left(\begin{array}{ccc}
1 & -\frac{3 g}{10 e} & -\frac{9 g}{10 e} \\
-\frac{3 g}{10 e} & \frac{9 g^{2}}{10 e^{2}} & 0 \\
-\frac{9 g}{10 e} & 0 & \frac{9 g^{2}}{10 e^{2}}
\end{array}\right), \quad \operatorname{det}(N)=0
$$

Remarkably, $N \sim m^{2}$. Simple order-of-magnitude considerations indicate that $\zeta \sim \alpha \tau^{-1} \sim 1 \mathrm{MeV}$, taking the time of formation of pseudoscalar condensate $\tau=1 \mathrm{fm}$ and the value of condensate of order of $f_{\pi}$.

For isotriplet pseudoscalar background $e^{2} \hat{\zeta}=3 \zeta \tau_{3}$, and the corresponding CS matrix takes the form

$$
N_{a b}^{\pi} \simeq\left(\begin{array}{ccc}
1 & -\frac{3 g}{2 e} & -\frac{g}{2 e} \\
-\frac{3 g}{2 e} & 0 & \frac{3 g^{2}}{2 e^{2}} \\
-\frac{g}{2 e} & \frac{3 g^{2}}{2 e^{2}} & 0
\end{array}\right), \quad \operatorname{det}\left(N^{\pi}\right)=0 .
$$

The VMD coefficients in (4),(5) are obtained from the anomalous Wess-Zumino action [19] and related to the phenomenology of radiative decays of vector mesons [20]. The ratios of matrix elements for isotriplet condensate in (5) are in direct agreement with the experimental decay constants for the processes $\pi_{0} \rightarrow \gamma \gamma, \quad \omega \rightarrow \pi_{0} \gamma, \quad \rho_{0} \rightarrow$ $\pi_{0} \gamma$ [19] and for the decay $\omega \rightarrow \pi \pi \pi$ [21] taken from [22]. Likewise the elements in (4) can be, in principle, estimated from the decays $\eta \rightarrow \gamma \gamma, \quad \eta^{\prime} \rightarrow \gamma \gamma, \quad \omega \rightarrow$ $\eta \gamma, \quad \rho_{0} \rightarrow \eta \gamma$. However, phenomenologically there exists a strong $\eta_{8}-\eta_{0}$ mixing effect finally resolved in the
$\mathrm{SU}(3)$ flavor scheme [23]. Only the ratio of the decay widths $\omega \rightarrow \eta \gamma, \quad \rho_{0} \rightarrow \eta \gamma$ is little sensitive to the mixing and confirms the off-diagonal elements of (4). In this letter we ignore the above mixing and restrict ourselves to $\mathrm{SU}(2)$.

The mass-shell equations for vector mesons read

$$
\begin{aligned}
& K_{a b}^{\mu \nu} V_{\nu, b}=0 ; \quad k^{\nu} V_{\nu, b}=0, \\
& K^{\mu \nu} \equiv g^{\mu \nu}\left(k^{2} \mathbf{I}-m^{2}\right)-k^{\mu} k^{\nu} \mathbf{I}-i \varepsilon^{\mu \nu \rho \sigma} \zeta_{\rho} k_{\sigma} N,(6)
\end{aligned}
$$

selecting out three physical polarizations for massive vector fields. In fact, these three polarizations contribute into the vector field propagators as they couple to conserved fermion currents. The longitudinal polarization $\varepsilon_{L}^{\mu}$ is orthogonal to $k_{\mu}$ and to the CS vector $\zeta_{\mu}$

$$
\varepsilon_{L}^{\mu}=\frac{\zeta^{\mu} k^{2}-k^{\mu}(\zeta \cdot k)}{\sqrt{k^{2}\left((\zeta \cdot k)^{2}-\zeta^{2} k^{2}\right)}}, \quad \varepsilon_{L} \cdot \varepsilon_{L}=-1,
$$

for $k^{2}>0$. The mass of this state remains undistorted. The transversal (circular) polarizations $\varepsilon_{ \pm}^{\mu}$ on the other hand satisfy

$$
K_{\nu}^{\mu} \varepsilon_{ \pm}^{\nu}=\left(k^{2} \mathbf{I}-m^{2} \pm \sqrt{(\zeta \cdot k)^{2}-\zeta^{2} k^{2}} N\right) \varepsilon_{ \pm}^{\mu} .
$$

As previously mentioned we restrict ourselves to an isosinglet pseudoscalar background $a(t)$. The spectrum can be found after the simultaneous diagonalization of matrices $m^{2}, N$ and particularizing to the case $\zeta_{\mu} \simeq$ $(\zeta, 0,0,0)$

$$
\begin{aligned}
& N=\operatorname{diag}\left[0, \frac{9 g^{2}}{10 e^{2}}, \frac{9 g^{2}}{10 e^{2}}+1\right] \sim \operatorname{diag}[0,1,1] \\
& m^{2}=m_{V}^{2} \operatorname{diag}\left[0,1,1+\frac{10 e^{2}}{9 g^{2}}\right] \sim \operatorname{diag}[0,1,1]
\end{aligned}
$$

namely

$$
k_{0}^{2}-\vec{k}^{2}=m_{V}^{2} \pm \frac{9 g^{2}}{10 e^{2}} \zeta|\vec{k}| \simeq m_{V}^{2} \pm 360 \zeta|\vec{k}| \equiv m_{V, \pm}^{2} .
$$

Thus in the case of isosinglet pseudoscalar background the massless photons are not distorted when mixed with massive vector mesons. In turn, massive vector mesons split into three polarizations with masses $m_{V,-}^{2}<m_{V, L}^{2}<$ $m_{V,+}^{2}$. This splitting unambiguously signifies local parity breaking as well as breaking of Lorentz invariance due to the time-dependent background. For large enough $|\vec{k}| \geq 10 e^{2} m_{V}^{2} / 9 g^{2} \zeta \simeq m_{V}^{2} / 360 \zeta$ vector meson states with negative polarization become tachyons. However their group velocity remains less than the light velocity 24] provided that $\zeta<20 e^{2} m_{V} / 9 g^{2} \simeq m_{V} / 180 \approx 4.3 \mathrm{MeV}$. For higher values of $\zeta$ the vacuum state becomes unstable, namely, polarization effects give an imaginary part for the vacuum energy. Note that the position of resonance poles for \pm polarized mesons is moving with wave vector $|\vec{k}|$ and therefore they reveal themselves as "giant" 
resonances. The enlargement of the resonant region potentially leads to a substantial enhancement of their contribution to dilepton production away from their nominal vacuum resonance position.

The production rate of dileptons pairs mediated by $\rho$ mesons takes a form similar to the one given in [7] but with modified propagators due to LPB, according to our previous discussion

$$
\begin{aligned}
& \frac{d N}{d^{4} x d M} \simeq c_{\rho} \frac{\alpha^{2} \Gamma_{\rho} m_{\rho}^{2}}{3 \pi^{2} g^{2} M^{2}}\left(\frac{M^{2}-4 m_{\pi}^{2}}{m_{\rho}^{2}-4 m_{\pi}^{2}}\right)^{3 / 2} \Theta\left(M^{2}-4 m_{\pi}^{2}\right) \\
& \times \sum_{\epsilon} \int_{M}^{\infty} d k_{0} \frac{\sqrt{k_{0}^{2}-M^{2}}}{e^{k_{0} / T}-1} \frac{m_{\rho, \epsilon}^{4}\left(1+\frac{\Gamma_{\rho}^{2}}{m_{\rho}^{2}}\right)}{\left(M^{2}-m_{\rho, \epsilon}^{2}\right)^{2}+m_{\rho, \epsilon}^{4} \frac{\Gamma_{\rho}^{2}}{m_{\rho}^{2}}}
\end{aligned}
$$

Finite lepton mass corrections are not shown in (11) but included in the fits. For $\omega$ mesons a similar expression is used but without the two pion threshold, characteristic of the dominant coupling of the $\rho$ to hadronic matter via two-pion fusion. A simple thermal average has been included but we remark that the temperature $T$ is an effective one that may in fact depend on the range of $M$, $p_{T}$ and centrality. The coefficients $c_{\rho}, c_{\omega}$ parametrize in an effective way the total cross-sections for vector meson creation. Because they are not known with precision in the present setting, particularly their off-shell values, the relative weights are used as free parameters in the hadronic 'cocktail' [1, 25, 26]. This is the procedure used both by NA60 and PHENIX and we shall follow it here too. The usual 'cocktail' contains weights normalized to the peripheral collisions result (roughly agreeing with existing $p p$ and $p$-nucleus data). For semi-central and central collisions, particularly at low $p_{T}$ the $\rho / \omega$ ratio needs to be enhanced by a factor 1.6 in the case of NA60 [1] or approximately 1.8 in PHENIX [27].

A simple thermal average is appropriate only for central collisions and moderate values of $p_{T}$. No serious attempt will be made here to extrapolate to peripheral processes, but an appropriate mixture of distorted and non-distorded vector mesons should be adequate. Likewise to include a proper description of the $p_{T}$ spectrum a more realistic statistical description would be needed. Finally, for a proper comparison with the experimental results our expressions would have to be propagated through the experimental acceptances. Nevertheless the general features of the LPB-induced modifications should be already visible in a simplified description.

NA60 has obtained accurate results for the $\rho$ spectral function [1] by measuring the $\mu^{+} \mu^{-}$spectrum with unprecedent precision and by carefully subtracting the contributions from the 'cocktail' except the $\rho$ itself. The corresponding data for central collisions is shown in Fig. 1 along with the contribution from the 'cocktail' scaled by the 1.6 factor previously indicated and using $m_{\rho}=750$ $\mathrm{MeV}$. The 'cocktail' $\rho$ can be obtained from (111) by setting $\zeta=0$ and adjusting the effective temperature to $T \simeq 300 \mathrm{MeV}$ [26]. However, even after optimization
$\mathrm{dN} / \mathrm{dM}$ per $20 \mathrm{MeV}$

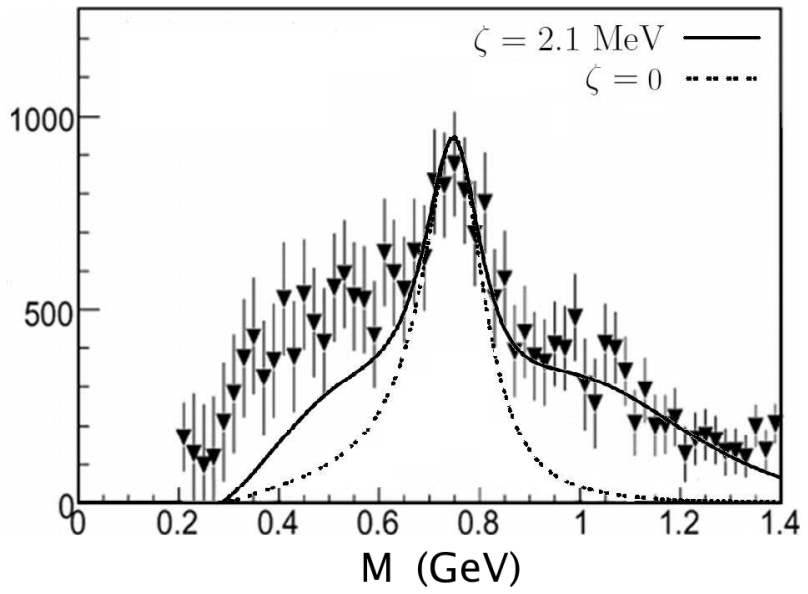

FIG. 1: The $\rho$ meson contribution into the dilepton production is shown for parity symmetric nuclear matter $\zeta=0$ and for local parity breaking with $\zeta=2 \mathrm{MeV}$ compared to the NA60 measurement for central collisons and all $p_{T}$. Both contributions are normalized to produce the same on-shell cross-section for $\rho$ meson production, which is fitted to the experiment. The effective temperature is $300 \mathrm{MeV}$.

of the constant to reproduce central $\rho$ production, the agreement of the data with the 'cocktail' $\rho$ is poor, reflecting the long standing problem of the insufficient dilepton yield. In the same figure we show, after adjusting the scale to reproduce the $\rho$ peak, the result for $\zeta \simeq 2 \mathrm{MeV}$. Clearly the agreement is much better, particularly on the right of the $\rho$ peak. On the left of the resonace there is a noticeable discrepancy for $M<650 \mathrm{MeV}$ approximately; experimental points seem to be shifted upwards by a small constant amount with respect to the prediction from LPB. It is revealing that the upper kinematical limit for the Dalitz process $\omega \rightarrow \mu^{+} \mu^{-} \pi^{0}$ is $643 \mathrm{MeV}$.

Important contributions to dilepton production at lower invariant masses are the Dalitz processes $\pi_{0} \rightarrow$ $\gamma e^{+} e^{-}, \eta \rightarrow \gamma l^{+} l^{-}$, nearly saturating the $l^{+} l^{-}$production in the $M<300 \mathrm{MeV}$ region, and $\omega \rightarrow l^{+} l^{-} \pi^{0}$. The latter has a partial lepton width nearly identical to the one of $\rho \rightarrow l^{+} l^{-}$and thus expected to show a similar behavior. The Kroll-Wada formula 28] includes the contribution of vector mesons to the previous Dalitz processes and it remains valid in the case of LPB provided that we replace the vector meson masses by the values in (10) according to the intermediate meson polarization $(L, \pm)$. We have checked that this contribution shows an enhancement but this along with all other hadronic processes relevant for dilepton production will be discussed in a separate publication. Indeed NA60 has been able to itentify the individual contributions from $\eta$ and $\omega$ Dalitz decays and found an enhancement, particularly for the latter 25].

Note that the $\rho$ mean free path $(1.3 \mathrm{fm})$ is much shorter than the expected size of the hadronic gas fireball $(L=5 \div 10 \mathrm{fm})$. The life-time of the fireball is compa- 
rable $\tau_{F B} \simeq L$. The time spent by a ultra-relativistic resonance of width $\Gamma$ inside the fireball is related to its mean free path $\sim 1 / \Gamma$ in such a medium. Therefore when $\Gamma \tau_{F B} \ll 1$ a tangible suppression of the resonant enhancement $1 / \Gamma^{2}$ arises. Around the distorted resonance peak a crude estimation of this suppression gives $\sim \tau_{F B}^{2} \Gamma^{2}$ in relative units. This suppression affects all long lived particles such as the ones entering the Dalitz processes for the LMR I, and it is also relevant to $\omega$ meson decays away from the vacuum peak. It is also the ultimate reason why it is unnecessary to include the $\phi$ vector resonance in the discussion. In our results, unless stated otherwise, we have not included any modification of the $\omega$ propagator due to LPB.

Moving now to the PHENIX $e^{+} e^{-}$data 2, 29] we observe that it has a much poorer precision and a numerical subtraction of the 'cocktail' to determine different individual contributions is meaningless. For low values of $p_{T}$ and for central collisions the effective temperature quoted by PHENIX lies in the region $100 \div 150 \mathrm{MeV}$ for the range of invariant masses under consideration. Based on the standard 'cocktail' we apply the normalization factor 1.8 already mentioned in order to approximately account for the correct $\rho / \omega$ ratio and use Eq. (11) with $\zeta \neq 0$ to include the effects of LPB. Fig. 2 shows the predicted $e^{+} e^{-}$yield for $z=1$ which is close to the optimal fit. The precision of the data does not allow for such clear cut conclusions as in the case of NA60 but it is clear that LPB noticeably improves the agreement to the data as compared to the 'cocktail'.

We would like to emphasize the extraordinary simplicity of the approach presented here. The fits presented use the values (effective temperatures, normalizations, etc. ) quoted by the experiment themselves. The only free parameter is $\zeta$, which is expected to depend on the characteristics of the collision. It should also be said clearly that the presence of LPB does not preclude other many body or in-medium corrections [7], as long as they do not represent double counting.

We now summarize the signatures and outline possible searches of local parity breaking.

Polarization: Dileptons produced for values of the invariant mass above and below the $\rho+\omega$ pole are predominantly of opposite circular polarizations. Thus one could search for a asymmetries among longitudinal and transverse polarization for different $M$ in event-by-event measurements. These measurements may reveal in an unambiguous way the existence of parity violation .

Distorted photons: At low energies massive vectors dominate the amplitudes but photons should also show a distortion induced by LPB. Those with '+' polarization exhibit different momentum thresholds $\sim 4 \mathrm{~m}_{l}^{2} / \zeta$ to show a resonant behavior for different dilepton species[17]. Note that finite size suppresion is relevant for photons.

The nature of the condensate: Mixing of photons with vector mesons is sensitive to isospin of pseudoscalar condensate and therefore the fraction of distorted photon decays helps to disentangle its isospin content.

\section{$\mathrm{dN} / \mathrm{dM}\left(\mathrm{GeV}^{-1}\right)$}

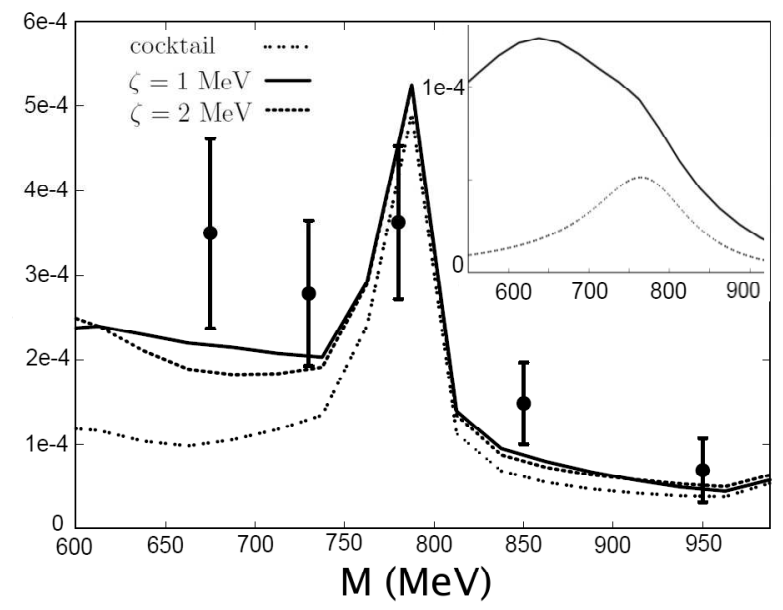

FIG. 2: The meson contribution to dilepton production is shown for parity symmetric nuclear matter $\zeta=0$ (discontinuous line) and for local parity breaking with $\zeta=1,2 \mathrm{MeV}$ (solid line and dotted line, respectively) compared to the PHENIX measurements for minimum bias, all $p_{T}$ events. The $\zeta=0$ line is just the 'cocktail' contribution as quoted by PHENIX [29]. The $\zeta \neq 0$ results from enhancing the $\rho / \omega$ ratio by a factor 1.8 without changing the $\omega$ normalization w.r.t. the hadronic 'cocktail'. The $\omega$ contribution itself includes a $20 \%$ of LPB contribution $(\zeta=1)$ and $80 \%$ pure 'cocktail' $(\zeta=0)$ to partially account for medium effects. A reasonable fit is obtained for $T=110 \mathrm{MeV}$ (compatible with PHENIX estimates for low $\left.p_{T}\right)$ and $\zeta=1 \mathrm{MeV}$. Data for $M<650 \mathrm{MeV}$ is not shown as the contribution from the Dalitz processes has not been included. Inset: the rho spectral function for $\zeta=1 \mathrm{MeV}$ is compared to the cocktail one with the same assumptions.

To summarize, in a time-dependent pseudoscalar background massless photons of ' + ' polarization and massive vector mesons behave as giant resonances after averaging over thermal distribution. For an isosinglet pseudoscalar background in the framework of VMD only the massive vector mesons $\rho$ and $\omega$ propagators are distorted due to mixing. We have computed their contribution and found that they naturally tend to produce an overabundance of dilepton pairs in the $\rho+\omega$ resonance region. The modified $\rho$ spectral function shows features very similar to the ones measured by NA60 in dimuon events. The PHENIX data is much better described by this mechanism than by adjusting the standard hadronic 'cocktail' in the $\rho+\omega$ resonance region. At lower invariant masses the Dalitz processes $\pi^{0} \rightarrow \gamma e^{+} e^{-}, \eta \rightarrow \gamma l^{+} l^{-}$and $\omega \rightarrow l^{+} l^{-} \pi^{0}$ saturate the hadronic contribution to $e^{+} e^{-}$production and are enhanced by the LPB-induced modifications on the vector meson propagators; detailed results are deferred to a future publication. The only free parameter is $\zeta$, characterizing the time variation of the pseudoscalar condensate. A good fit to the data is obtained for natural values of $\zeta$. The possibility that the LPB condensate is an isotriplet or an admixture of isotriplet and isosinglet has been discussed. Experimental signals of the manifes- 
tation of LPB in heavy ion collisions have been suggested. Thus local parity breaking seems capable of explaining in a natural way the PHENIX/CERES/NA60 'anomaly' and searching for its manifestation in dilepton production represents an interesting challenge for some of the LHC collaborations.

We acknowledge the financial support from projects
FPA2007-66665, 2009SGR502, CPAN (Consolider CSD2007-00042) and FLAVIANET. A.\& V. Andrianov are supported also by Grants RFBR 09-02-00073-a and 10-02-00881-a and by SPbSU grant 11.0.64.2010. We thank D. d'Enterria and J. Casalderrey for discussions and useful remarks.
[1] M. Masera, [HELIOS/3 Collaboration], Nucl. Phys. A 590, 103c (1995); G. Agakichiev et al.[CERES Collaboration], Eur. Phys. J. C4, 231 (1998); Phys. Lett. B 690, (2010); R. Arnaldi et al.[NA60 Collaboration], Phys. Rev. Lett. 96, 162302 (2006).

[2] A. Adare et al.[PHENIX Collaboration], Phys. Rev. C81, 034911 (2010).

[3] K. O. Lapidus and V. M. Emel'yanov, Phys. Part. Nucl. 40, 29 (2009).

[4] I.Tserruya, 0903.0415 [nucl-ex].

[5] K. O. Lapidus [HADES Collaboration], Phys. Atom. Nucl. 73, 985 (2010).

[6] G. E. Brown and M. Rho, Phys. Rev. Lett. 66, 2720 (1991).

[7] R. Rapp and J. Wambach, Adv. Nucl. Phys. 25, 1 (2000); W. Liu, R. Rapp, Nucl. Phys. A796, 101 (2007); H. van Hees and R. Rapp, Nucl. Phys. A806, 339 (2008).

[8] W. Cassing and E. Bratkovskaya, Nucl. Phys. A807, 214 (2008); E. L. Bratkovskaya, W. Cassing and O. Linnyk, Phys. Lett. B670, 428 (2009).

[9] K. Dusling and I. Zahed, Nucl. Phys. A825, 212 (2009).

[10] H. van Hees and R. Rapp, Phys. Rev. Lett. 97, 102301 (2006).

[11] J. Ruppert et al, Phys. Rev. Lett. 100, 162301 (2008).

[12] D. Kharzeev, R. D. Pisarski and M. H. G. Tytgat, Phys. Rev. Lett. 81, 512 (1998); K. Buckley, T. Fugleberg, and A. Zhitnitsky, Phys. Rev. Lett. 84, 4814 (2000); D. Kharzeev, Phys.Lett. B633, 260 (2006); D. E. Kharzeev, L. D. McLerran and H. J. Warringa, Nucl. Phys. A803, 227 (2008).

[13] P. Buividovich, M. Chernodub, E. Luschevskaya and M. Polikarpov, Phys. Rev. D 80, 054503 (2009).

[14] B. I. Abelev et al. [STAR Collaboration], Phys. Rev. Lett. 103, 251601 (2009); S. A. Voloshin, J. Phys. Conf. Ser.
230, 012021 (2010).

[15] A. Gorsky and M. B. Voloshin, 1006.5423 [hep-th].

[16] A. A. Andrianov and D. Espriu, Phys. Lett. B 663, 450 (2008); A. A. Andrianov, V. A. Andrianov and D. Espriu, Phys. Lett. B 678, 416 (2009).

[17] A. A. Andrianov, D. Espriu, P. Giacconi and R. Soldati, JHEP 0909, 057 (2009); A. A. Andrianov, D. Espriu, F. Mescia and A. Renau, Phys. Lett. B 684, 101 (2010).

[18] J.J. Sakurai, Ann. of Physics 11, 1 (1960); Currents and Mesons, (The University of Chicago Press, Chicago 1969).

[19] N. Kaiser and U.-G. Meissner, Nucl. Phys. A 519, 671 (1990); E. Truhlik, J. Smejkal and F.C. Khanna, Nuclear Physics A 689, 741 (2001).

[20] O. Dumbrais et al., Nucl. Phys. B 216, 277 (1983).

[21] F. Klingl, N. Kaiser and W. Weise, Z. Phys. A356, 193 (1996).

[22] K. Nakamura et al. [Particle Data Group], J. Phys. G 37, 075021 (2010).

[23] T. Feldmann, P. Kroll and B. Stech, Phys. Rev. D58, 114006 (1998) ; Y. N. Klopot, A. G. Oganesian and O. V. Teryaev, arXiv:0911.0180 [hep-ph].

[24] J. Alfaro, A. A. Andrianov, M. Cambiaso, P. Giacconi and R. Soldati, Int. J. Mod. Phys. A 25, 3271 (2010).

[25] R. Arnaldi et al [NA60 Collaboration], Phys. Lett. B 677, 260 (2009)

[26] R. Arnaldi et al. [NA60 Collaboration], Eur. Phys. J. C 61, 711 (2009).

[27] V.L. Rykov, private communication.

[28] L. G. Landsberg, Phys. Rep. 128, 301 (1985).

[29] Numerical data extracted from http://www.phenix.bnl.gov/phenix/WWW/info/data/ ppg088_data.html/fig26.txt 